\title{
Robot Control: From Silicon Circuitry to Cells
}

\author{
Soichiro Tsuda ${ }^{1, \star}$, Klaus-Peter Zauner ${ }^{2}$, and Yukio-Pegio Gunji ${ }^{1}$ \\ 1 Graduate School of Science and Technology, \\ Kobe University Nada, Kobe 657-8501, Japan \\ Fax: +81-78-803-5759 \\ 026d874n@y04.kobe-u.ac.jp, yukio@kobe-u.ac.jp \\ 2 School of Electronics and Computer Science, \\ University of Southampton, SO17 1BJ, United Kingdom \\ Fax: +44-23-8059-2865 \\ kpz@ecs.soton.ac.uk
}

\begin{abstract}
Life-like adaptive behaviour is so far an illusive goal in robot control. A capability to act successfully in a complex, ambiguous, and harsh environment would vastly increase the application domain of robotic devices. Established methods for robot control run up against a complexity barrier, yet living organisms amply demonstrate that this barrier is not a fundamental limitation. To gain an understanding of how the nimble behaviour of organisms can be duplicated in made-for-purpose devices we are exploring the use of biological cells in robot control. This paper describes an experimental setup that interfaces an amoeboid plasmodium of Physarum polycephalum with an omnidirectional hexapod robot to realise an interaction loop between environment and plasticity in control. Through this bio-electronic hybrid architecture the continuous negotiation process between local intracellular reconfiguration on the micro-physical scale and global behaviour of the cell in a macroscale environment can be studied in a device setting.
\end{abstract}

\section{The Biological Paradigm}

Information processing is essential for life. From the very outset living matter had to defend its organisation against the onslaught of entropy. Subsequently the need to compete with rivalling life forms required evermore refined information processing. As a consequence organisms exhibit an intriguing sophistication in overcoming computationally difficult challenges. In the area of robotics, where restrictions in power consumption and size collide with real-time processing requirements for complex data streams, the discrepancy between technology and nature is particularly apparent.

Although competent artificial autonomous systems capable of successfully acting in an unknown and unbounded dynamic environment are not in sight, directing attention on biological systems has brought several issues to the fore.

\footnotetext{
* Corresponding author.
} 
It is well known that organisms employ a pragmatic tailoring of sensory information for their specific needs [1. Conversely, it has been shown that apparently complex behaviour requires only relatively simple control structures [2]. Central to a reduction in computational requirements for control is the task specific adaptation of sensors and actuators [3. In fact the conceptual separation between sensing, coordinating, and acting may not be tenable for such systems. Mechanical components of a robot's structure can become part of the information processing architecture [4] and thus behaviour is not reflecting the direct actions of a controller but emerges from the interaction of control, body and environment [5, 6].

After what has been stated it may seem that such an approach achieves efficiency by trading away versatility. Yet, clearly, organisms exhibit an enviable capability for coping with unknown situations. How can they achieve efficiency without compromising versatility? Arguably, the key lies in the plasticity of the architecture that allows for the dynamic formation of sensory, computational, and effector structures in reciprocation with the environment [7, 8].

The paradigms outlined above have in common that they go beyond the remit of formal computation by drawing on specific properties of the computing substrate and even extending the latter to include the environment of the robotic device. Hybrid architectures that interface nature's computing substrates with artificial devices open up a path to investigate these paradigms. In the present context the coupling of biological tissue or whole organisms with robotic devices is of interest. Some of the research in this area is motivated by potential applications in prosthetics. It has been demonstrated, for instance, that simultaneous recordings from populations of neurons can enable rats to control a robotic arm 9]. Other approaches focus on the the computational properties of the neural tissue. DeMarse et al. have interfaced cultured rat neurons with a computer simulated environment to study the effect of closed loop feedback to the neuronal network [10]. In a similar vein Reger et al. extracted the brain stem of a fish larva and stimulated it with signals derived from light sensors mounted on a mobile robot. Neural activity in two regions of the brain stem was used to control the robot's movements. We must not omit to mention here the art installations of Ken Rinaldo. He built a number of different robots that are controlled by the position to which a fish swims in its tank and he has implemented what is likely to be the first interaction among robots controlled by organisms (other than humans) [1].

The direction of the work presented in this paper differs from the aforementioned in its focus on robot technology. Here the robot does not serve as a tool for the organism that is in control, nor is it purely a research tool in the study of biological information processing. The long-term objective of the line of research described here is the integration of, potentially modified, cells into made-for-purpose robotic devices.

In the following we first consider the self-organisation and information processing properties of single cells, next focus on a specific cell, the plasmodia of Physarum polycephalum, and then describe a hybrid approach in which a 
plasmodium is integrated into a robot controller to import the plasticity and adaptability of a living organism into a device architecture.

\section{Cellular Information Processing}

Single cells cannot take recourse to specialised tissues such as a nervous system for their information processing needs. Nonetheless, even bacteria possess elaborate signal processing capabilities 12. Single cell organisms, by necessity, implement sensors, information processing, and effectors on the molecular level. In examining what is known about nature's molecular level computing it becomes evident that matter is used in a markedly different way than in conventional computing architectures. Information processing mechanisms are tightly coupled to physiochemical properties of the materials rather than being narrowly constraint to enact a rigid formalism [13].

Rothstein speculated that living matter has a large amount of information coded in its structure and used the term 'instructions' for the constraints the system structure imposes on the physicochemical dynamics [14]. This viewpoint is supported by experimental findings. If cells are cooled to very low temperatures all molecules in the cell come almost to a halt. During warming the linear and angular momenta of the molecules in the cell are randomised. This procedure erases the dynamic state information of the cell. Nevertheless cells survive and the cell's static structure is able to revive the dynamics of the living state after warming [15. Instructions in the sense of Rothstein, however, should not be misconstrued with the careful state preparation required in programming conventional computing devices which depend crucially on the initial state for correct operation. On the contrary, indeed, it is found, for instance, that physicochemical dynamics is able to recreate the intracellular infrastructure even after the spatial arrangement of practically all enzymes has significantly been altered by ultracentrifugation [16]. In combination these observations point to an organisational principle in which cell structure and cell dynamics mutually maintain each other. Prerequisite for such an organisation is, what may be called structure-function self-consistency. Not only does the structure self-organise, as for example in protein folding or virus self-assembly, but also the functional dynamics arises directly from the physical interaction of the components following a course determined by free-energy minimisation. This principle is very different from established information processing technology where the course of a computation is critically dependent on the precise preparation of an initial machine state. It does not only contribute to the robustness and resilience of natural information processors, but, moreover, allows for more efficient implementations. This is the case, because a smaller fraction of the architecture is required to establish constraints and the system state does not need to be tightly constraint with high energy barriers [17].

We will now turn to the the plasmodium of the true slime mold Physarum polycephalum, to discuss a specific example of a self-organising, robust information processing architecture that exhibits the aforementioned properties. 


\section{Characteristics of Physarum Plasmodia}

The plasmodial stage of Physarum polycephalum is a single, giant, multi-nuclear cell. It moves like a large amoeba and is feeding on bacteria. The size of this amorphous organism ranges from several tens of micrometers to a few meters. Figure 1 shows the typical organisation of cytoplasm in a plasmodium. An external membrane encloses a possibly large mass of cytoplasm. Small cells often rely on diffusion to communicate materials and signals internal to the cell. For the giant cells of Physarum polycephalum, however, diffusion among distant parts of the cell would be exceedingly slow. An active mechanism is therefore required to coordinate behaviour across the whole organism. A network of tubes spans the plasmodium and connects to its flat border zone. Cytoplasm oscillates forward and backward within the tubes. This, so called shuttle streaming, is driven by hydrostatic pressure gradients along the tubes. The tubes themselves apparently consist of gelled cytoplasm and tubes are formed, interconnected, resized, and disassembled to adapt to changes in the environment as well as to growth [18].

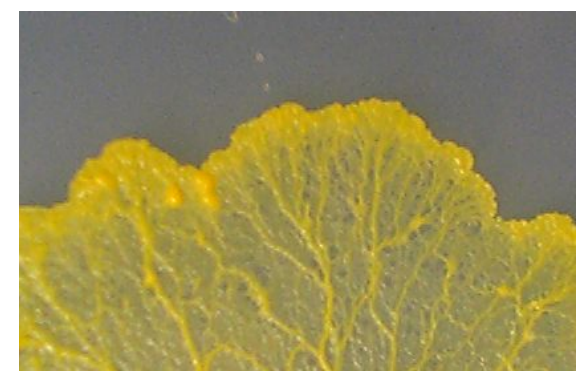

Fig. 1. Part of a plasmodium of Physarum polycephalum in developed form. Sheet-like fringe areas (upper part of the photograph) are connected by a network of tubular structures (lower part of the photograph). The section shown is $30 \mathrm{~mm}$ wide.

\subsection{Distributed Information Processing}

The dynamic tubular network provides the necessary infrastructure to integrate information from peripheral zones of the plasmodium and enables this primitive motile system to respond to local stimuli with a coordinated movement of the whole organism. If the plasmodium locally touches a food source, it will gather around it and than cover it. Conversely if an area of the plasmodium comes into contact with a chemical it finds repulsive $(\mathrm{NaCl}$ for instance), the entire plasmodium will collectively move away from the stimulated region.

The plasmodium shows chemotaxis, phototaxis, thermotaxis and prefers humid over dry locations. Yet, it does not possess a central processing system for sensory signals. It relies on distributed information processing and communication through the shuttle streaming mechanism to generate the appropriate response to stimuli. The rhythm of the shuttle streaming is known to be synchronised with intracellular chemical oscillation, such as ATP and $\mathrm{Ca}^{2+}$ concentration [19]. A local attractive stimulus (e.g., glucose or warmth) is first converted 
to an increase in frequency of the local protoplasm oscillations and similarly a repellent (e.g., blue light) will lead to a reduced local frequency. The local oscillations are then communicated through the tubular network to couple to oscillations modulated by stimuli received at other regions of the cell [20]. Finally the overall oscillatory pattern of the cytoplasmic streaming gives rise to an approaching or escaping movement. This signal processing mechanism has been modelled with a reaction-diffusion system [21] and shown to be size-invariant [22].

Numerous observations have confirmed the versatility of the distributed processing implemented by the Physarum polycephalum plasmodium. Nakagaki and coworkers, for example, showed that the plasmodium can find the shortest path in a maze [23] and that it can solve small instances of optimisation problems [24]. Aono recently constructed a novel neural network system driven by a plasmodium [25. Some of us implemented self-repairing logic gates with plasmodia [26, which leads to another aspect of Physarum polycephalum that is of interest with regard to robot control.

\subsection{Self-repair and Robust Behaviour}

Among the most enviable features of natural information processing systems is their robustness. Typically they exhibit both, robust function in face of component variation or failure, and robust behaviour in face of unanticipated or paradoxical situations. Both are difficult to achieve with established robot control technology. With progressing miniaturisation of robotic devices these features will become increasingly important. The former because detailed prescriptive control in fabrication will become economically, if not physically, infeasible [27. and the latter because the potential application areas for miniaturised robotic devices call for a high degree of autonomy.

The self-maintenance principle of cells discussed in section 2 in combination with the distributed organisation described in the previous section endows the plasmodium with functional robustness. If a tube of a plasmodium is severed, cytoplasm pumped through the tube will leak into the area surrounding the cut and gel to seal the tube. This sol-gel state transition of the cytoplasm together with the fact that a plasmodium typically contains numerous nuclei allows for much more drastic damage to be overcome. A plasmoidum can be physically taken apart into small pieces, each of which will seal itself up and survive as a smaller, individual, fully functional plasmodium. But, moreover, if two plasmodia get in contact, they can fuse into a single cell and act as one plasmodium after the fusion. This feature has been exploited by Takamatsu et al. to construct coupled nonlinear oscillator circuits [28] and the robot control architecture described below builds on their technique.

As to the phenomena of behavioural robustness in the face of contradictory stimuli, Nomura observed that a plasmodium surrounded by a repellent chemical will eventually move towards the repulsive stimuli and break out of the trap [29]. Further studies are needed to establish whether this is the result of random errors in the information processing cascade that occur with low probability and thus, after a period of exploration, cause the escape, or whether a more sophisticated 
mechanism gives rise to the approach of the repellent. Studies with multiple attractants also indicate a graceful response of the plasmodium [24].

\subsection{Plasmodium Properties Used in the Robot Controller}

The cell-based robot controller described in the following section draws on many of the features mentioned so far. A few additional facts are pertinent to the construction and function of the bio-hybrid control architecture. Plasmodia can be grown in a desired geometry by means of a negative plastic mask. A diskshaped area with a diameter that is small compared to the spatial wavelength of the shuttle-streaming oscillations will be in synchrony and can be assumed to be a single oscillator. And, furthermore, if two such areas are connected by a channel in the mask, the phase wave of an oscillator is propagated only through tube structures formed in the channel [28].

The cytoplasm flow of the shuttle-streaming leads to oscillations in the local thickness of the plasmodium cell. Because plasmodia are not sensitive to light near $600 \mathrm{~nm}$ the shuttle-streaming can be monitored through the change in light absorbance concomitant with the thickness variation. To blue light, however, the plasmodia show negative phototaxis and blue or white light can therefore be used as stimuli [30]. In combination these two facts allow for an optical interface to the plasmodium.

\section{Robot Control with Physarum Circuits}

In contrast to conventional information processing architectures, the infrastructure of a cell is in a continual state of flow. This dynamic replacement of components serves maintenance and facilitates structural reorganisation. Selfmodification is thus inherent in cells. It would be very difficult to capture even part of this adaptive aspect in a purely artificial device based on current technology. By taking a hybrid approach that integrates a cell into a robot control architecture, however, we can experimentally investigate the interaction of a device that comprises autonomous, self-modifying, and self-maintaining components with its environment.

The design of the architecture is illustrated in Fig. 2. Local shuttle streaming oscillations of a plasmodium are measured and used to affect the phase and

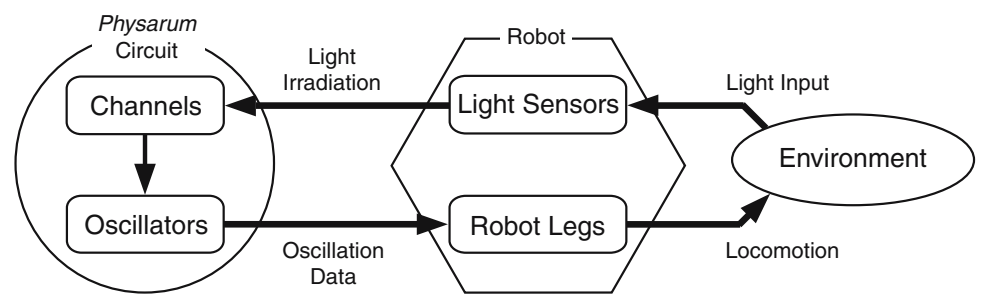

Fig. 2. Physarum-Robot-Environment Interaction Loop 
frequency of leg swing in a six-legged robot. The combined action of the legs results in a motion of the robot through its environment which is structured by light sources. Signals from light sensors mounted on the robot are transduced to white light stimuli for a plasmodium. These stimuli in turn affect the shuttle streaming and consequently alter the motion of the robot. However, the cell will respond also with structural reconfiguration. Conrad suggested that in such a self-modifying system structural improvements are possible if errors destabilise the structure and termed this process 'adaptive self-stabilisation' 31. We adapt a similar perspective, which briefly put, can be pictured as follows. A signal from the environment impinging on the cell gives rise to a local perturbation. As a consequence, locally, the cell state is not aligned with the global state of the cell. The cell will strive to reestablish a self-consistent state by updating its sensory, computational, and effector structures. These structures are of course highly interdependent and pursuing a self-consistent state may involve a lengthy cascade of self-modifications, a process here referred to as negotiation between the local and the global. From the structural changes that accompany the localglobal negotiation process, an altered global behaviour of the cell emerges. For a formal model of this process see 32 .

\subsection{The Making of a Cell Circuit}

To control the six legs of a hexapod robot we use the star shaped circuit shown in Fig[3. Each of the six circular wells acts as a nonlinear oscillator and all oscillators are coupled through the channels that meet at a single central point. The plasmodium is patterned by letting it grow into the open parts of a mask cut from plastic film that is placed on a $3-5 \mathrm{~mm}$ layer of $1.5 \%$ agar in a Petri dish. To fabricate the plastic mask, the circuit pattern with $\approx 1.5 \mathrm{~mm}$ diameter wells and $0.5 \mathrm{~mm}$ wide channels is printed with a laser printer on an overhead projector foil. The wells are cut out with a $1 / 16$ " hand punch (www.fiskars.com) and the channels are cut with a sharp blade under a stereomicroscope. The distance from the centre of a well to the junction of the six channels is $3.75 \mathrm{~mm}$.

When the mask is placed on the agar plate it leaves a moist agar region in the desired geometry exposed and confines this area with the dry plastic surface. The plasmodium preferentially grows on a moist surface and usually does not migrate over a dry region. The fact, above mentioned, that plasmodia can be cut into pieces and will fuse into a single cell allows for a cut-and-paste approach in filling the mask. Plasmodia are cultured on $1.5 \%$ agar gel plates that are sprinkled with oat flakes to supply bacteria as food for the plasmodium. A tip of a plasmodium culture is cut off and placed on the exposed agar gel inside each well so that it covers the well region (Fig. $3 \mathrm{~B}$ ). The Petri dish with the developing circuit is then incubated at about $20^{\circ} \mathrm{C}$ in the dark for 10 hours. During incubation the six plasmodia in the wells grow along the exposed agar surface in the channels (Fig. $3 \mathrm{C}$ ) and upon meeting in the centre fuse into a single plasmodium. Approximately four hours after fusion tube structures have been formed in the channels (Fig. 3D) and the circuit is ready for use. 


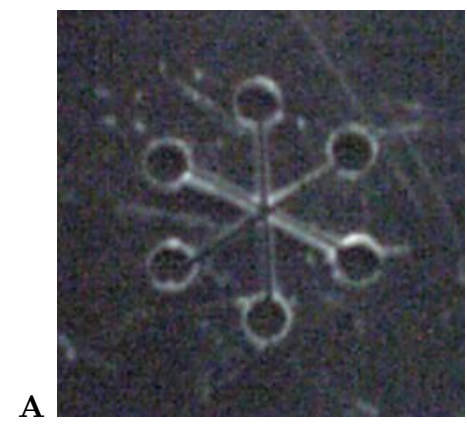

A

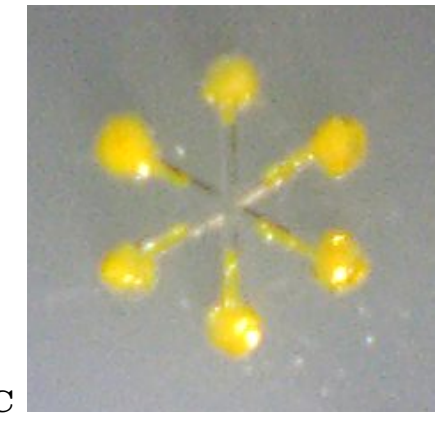

B

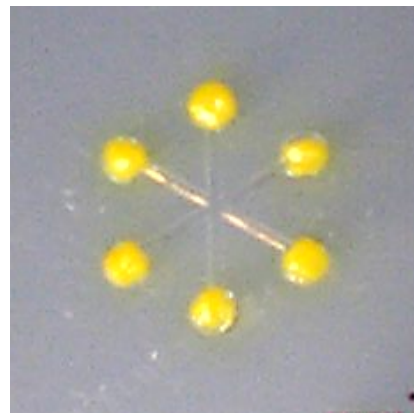

D

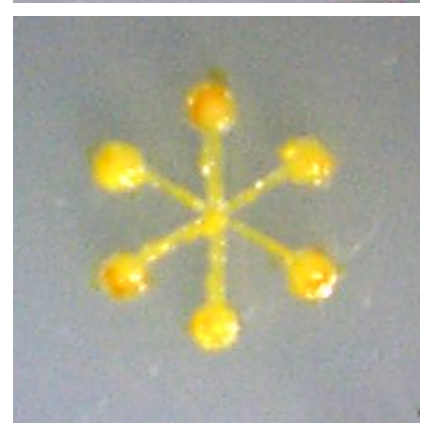

Fig. 3. Physarum circuit. The plasmodium is patterned as six oscillators with star coupling by means of a negative plastic mask (A). Panel (B) to (D) show snapshots of the plasmodia growth. Shortly after preparation (B), 5 hours after preparation (C), and the fully developed circuit 10 hours after preparation (D).

\subsection{The Robotic Platform and Cell-Robot Interface}

A simple six-legged robot with a hexagonal body and radial leg swing provides a platform with excellent static stability. There is no possibility for mechanical interference among the actuators, even if every leg performs random motions and on a flat surface the robot will not tip over. This robot, depicted in Fig. 4, can move and sense omnidirectional and offers numerous possible gate patterns with only a single degree of freedom per leg. If groups of legs are driven in anti-phase the robot exhibits directional movement. The legs of the robot are driven by six servos controlled through a serial interface with a PC. The robot is equipped with six light sensors that feed into six channels of a sound card in the PC. A flexible wire bundle connects the robot with the PC.

Now, to implement the interaction loop shown in Fig. 2, the robot needs to be bi-directionally interfaced with the Physarum circuit. The thickness variation of the plasmodium on the one side and its phototactic response on the other side allow for an all-optical interface. Figure [5] shows the overall architecture, the software for which was implemented in Tcl/Tk (cf., wiki.tcl.tk) using the Snack library (www.speech.kth.se/snack/).

One direction will transmit signals received by the robot's sensors to the plasmodium. The signals that arrive at the sound card of the PC are interpreted 

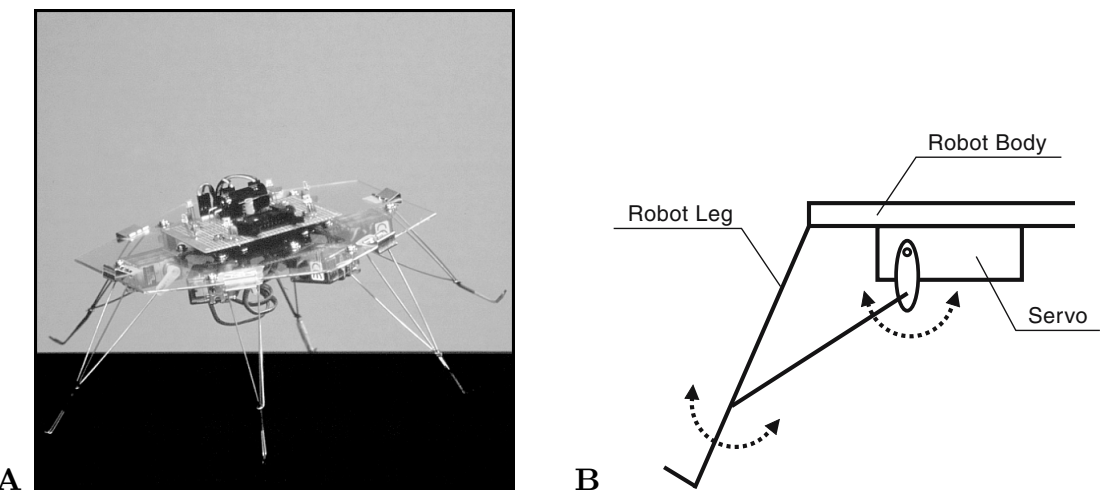

Fig. 4. A tethered hexapod robot (A) is used for its stance stability and gait flexibility. Each leg has only one degree of freedom and swings radial to the body (B). Whether a leg is in contact with the ground during its move will depend on the position of the other legs.

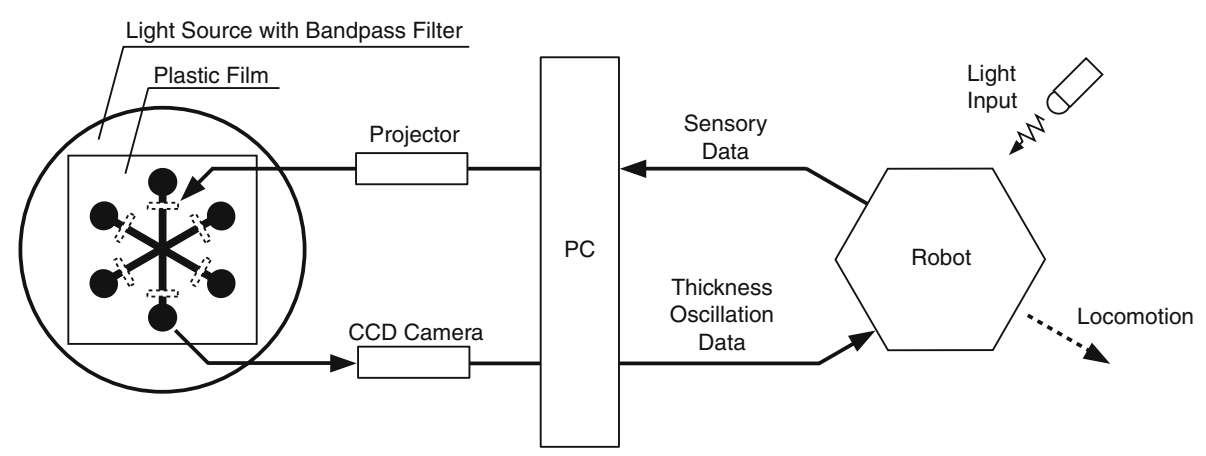

Fig. 5. Cellular robot controller. See text for details.

by the PC and translated into an entry from a fixed image table. Each image in the table has a different white pattern on black background. The image selected from the table is projected with a data projector onto the Physarum circuit in such a way that the white patterns (indicated by dashed rectangular boxes in the left of Fig. 51) fall on the channels that connect the wells in the circuit and thus locally stimulate the circuit. Each light sensor on the robot has a corresponding white bar in the projected pattern.

The other direction of the cell-robot interface will transmit the response of the plasmodium to the actuators of the robot. To this end the Petri dish with the fully developed circuit is placed on a cold light source which is bandpass filtered (NT46-152, www.edmundoptics.com) to the spectral region near $600 \mathrm{~nm}$. A CCD camera mounted above the Petri dish detects the red light transmitted through the Physarum circuit. For each well the 8-bit brightness values of pixels from the central region are averaged and used to calculate an amplitude signal for 
the oscillator comprised by the part of the plasmodium in that well. To suppress camera noise, a moving average over a window of 15 samples is used as final output signal of the well. The signal from the six oscillators typically have a period of one to two minutes - too long to be ideal for directly driving the legs of the robot. The plasmodial oscillators, however can be coupled to software oscillators that drive the six robot legs at a higher frequency, yet preserve the phase relationship of the physarum circuit wells. This form of coupling also compensates for the typical variations in amplitude of the signals measured from different wells. The phase relationships among the six legs determine the locomotion of the robot and as a consequence lead to changes in the light levels received by the robot's sensors and accordingly the white light stimulation received by the circuit - thus closing the interaction loop.

\subsection{Physarum Oscillatory Behaviour}

Experiments with a model of coupled nonlinear oscillators confirmed that the robotic platform is capable of directed motion and of direction change when the legs are driven by the oscillators. These experiments showed that changes in the phase relationship among the legs are sufficient to switch between different gait modes.

Our current experiments focus therefore on the phase relationship of the wells in the six-oscillator configuration described in section 4.1, The plasmoidum is known to be active even without food over several days and will go into a dormant state if starved longer. Measurements over extended time periods are therefore possible. We generally conduct experiments with the circuits for 5-10 hours. A typical behaviour of this circuit is shown in Fig. 6. The transitions among phase patterns that are visible in the figure are spontaneous, no stimulus has been applied over the time period depicted.

Stable global in-phase, anti-phase, and rotational oscillations have been observed. Brief periods of unstable oscillations also occur. These oscillations are inherent to the physiology of the plasmodium and occur even without external stimulation. Preliminary experiments with light stimulation indicate that local stimuli can affect the global circuit mode. For instance, we have observed two oscillators located directly opposite each other change from in-phase to anti-phase oscillation immediately after a light stimulus was removed. At present, however, we do not have enough data to establish the nature of this phenomenon. To further investigate the effect of local stimuli on the global oscillation patterns, we have recently modified the experimental procedure to also irradiate the wells with white light (interrupting the stimulus briefly to permit the measurement of transmitted red light).

As noted above, anti-phase oscillations are effective to generate one-directional movement of the robot. Conversely unstable oscillatory patterns typically result in a change of orientation of the robot. As a consequence, despite the robot's morphological symmetry, it will move in a different direction after a phase of unstable oscillations, even if the plasmodium returns to the previous anti-phase pattern. 


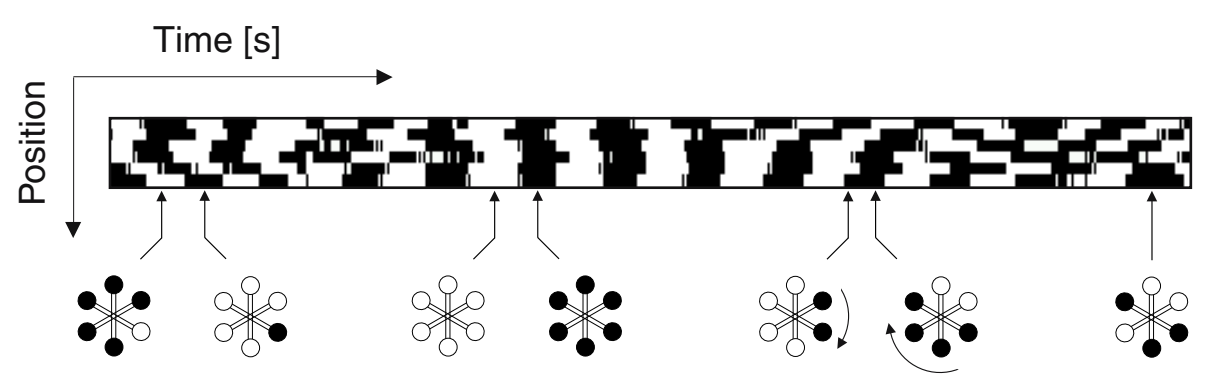

Fig. 6. Spontaneous change in phase relation among the six oscillators of the circuit shown in Fig 3 . The graph represents the binarised thickness oscillation rhythm for the oscillators. No stimulus was applied. The six horizontal bands from top to bottom correspond to oscillator wells 1-6, numbered counterclockwise from the upper right (one-a-clock) position. Black lines represent an increase and white vertical lines a decrease in thickness. The horizontal axis represents time with the total length of the graph corresponding to 1000s. Several spontaneous transitions among synchronisation patterns are observed over this time period.

\section{Discussion and Perspective}

Purposeful operation in a complex ambiguous environment requires versatility rather than controllability. With the current information technology paradigm finding more complex or efficient behavioural strategies is paraphrased as a problem of proper tuning of system parameters and components. But for a system that is required to operate in an ill-defined real world environment, the established concept does not work. The set of key parameters to be considered is bound to be vague and one cannot predetermine all of the possibilities at one's disposal. Nature evolved information processors that are up to this challenge. The plasticity that is a prerequisite for evolutionary progress at the same time opens a path to continual self-adaptation. The architecture of such a system is a response to the current and past interaction with the environment and does in general not have a fixed organisation. The system structure is in a perpetual state of change while local alterations to sensors and signal processing cascades affect the global behaviour which in turn requires a re-alignment of local structure. This local-global negotiation process replaces the tuning of parameters in a conventional system which is necessarily as much ad hoc as it is a priori. This crucial difference between natural and artificial information processors has received considerable attention in the theoretical literature (for pointers see the citations in sections 1, 2, and 4). An experimental approach from an information processing perspective, however, has been difficult.

Although our experiments are at an early stage, we expect that the biohybrid architecture presented above will open a path in this direction. From our experience so far, we conclude that the plasmodium of Physarum polycephalum is well suited to study device architectures based on autonomous components. At this stage many questions are open. We currently study the effect of the light 
input signals on circuits to gain a better understanding of what the determinants of the observed transitions in the phase patterns are. We are also investigating how the repertoire of phase patterns relates to circuit topology.

On a longer perspective, we expect robust biological cells such as those of molds and thermophilic bacteria to become an integral part of technological devices. We believe that the efficient and quality controlled nano-fabrication offered by biological cells may turn out to be a suitable way of obtaining highly integrated, robust information processors, at least for niche applications.

\section{Acknowledgements}

The authors thank Masashi Aono for helpful discussions. The research reported here was supported in part by the Science and Technology Agency of Japan through the Center of Excellence (COE) program.

\section{References}

1. J. Y. Lettvin, H. R. Maturana, W. S. McCulloch, and W. H. Pitts. What the frog's eye tells the frog's brain. Proc. Inst. Radio Engr., 47:1940-1951, 1959. Reprinted in The Mind: Biological Approaches to its Functions, W. C. Corning and M. Balaban, (Eds.), 1968, pp. 233-258.

2. V. Braitenberg. Experiments in Synthetic Psychology. MIT Press, Cambridge, MA, 1984.

3. B. Webb. View from the boundary. Biological Bulletin, 200(2):184-189, 2001.

4. B. Hasslacher and M. W. Tilden. Living machines. Robotics and Autonomous Systems, pages 143-169, 1995.

5. R. D. Beer. Intelligence as Adaptive Behavior, an Experiment in Computational Neuroethology. Academic Press, 1990.

6. H. J. Chiel and R. D. Beer. The brain has a body: adaptive behavior emerges from interaction of nervous system, body and environment. Trends In Neuroscience, 12:553-557, 1997.

7. H. R. Maturana and F. J. Varela. Autopoiesis and Cognition: The Realization of the Living, volume 42 of Boston Studies in the Philosophy of Science. D. Reidel Publishing, Dordecht, Holland, 1980.

8. P. Cariani. Some epistemological implications of devices which construct their own sensors and effectors. In F. J. Varela and P. Bourgine, editors, Toward a Practice of Autonomous Systems: Proceedings of the First European Conference on Artificial Life, pages 484-493, Cambridge, MA, 1992. MIT Press.

9. J. K. Chapin, K. A. Moxon, R. S. Markowitz, and M. A. L. Nicolelis. Real-time control of a robot arm using simultaneously recorded neurons in the motor cortex. Nature neuroscience, 2(7):664-670, 1999.

10. T. B. DeMarse, D. A. Wagenaar, A. W. Blau, and S. M. Potter. The neurally controlled animat: Biological brains acting with simulated bodies. Autonomous Robots, 11:305-310, 2001.

11. K. Rinaldo. Augmented fish reality. ARS Electronica Center, Linz, 2004. See also: http://accad.osu.edu/ ${ }^{\sim}$ rinaldo/works/augmented/.

12. J. Adler and W.-W. Tso. "Decision"-making in bacteria: Chemotactic response of Escherichia coli to conflicting stimuli. Science, 184:1292-1294, 1974. 
13. K.-P. Zauner. Molecular information technology. Critical Reviews in Solid State and Material Sciences, 30(1):33-69, 2005.

14. J. Rothstein. Information, measurement, and quantum mechanics. Reprinted in: H. S. Leff and A. F. Rex (Eds.), Maxwell's Demon-Entropy, Information, Computing, pp. 104-108, Bristol: Adam Hilger, 1990., 1951.

15. A. I. Skoultchi and H. J. Morowitz. Information storage and survival of biological systems at temperatures near absolute zero. Yale J. Biol. Med., 37:158-163, 1964.

16. H. Kondo, M. Yamamoto, and M. Watanabe. Reversible intracellular displacement of the cytoskeletal protein and organelles by ultracentrifugation of the sympathetic ganglion. J. Submicrosc. Cytol. Pathol., 24:241-250, 1992.

17. Michael Conrad. The price of programmability. The Universal Turing Machine: A Half-Century Survey, pages 285-307, 1988.

18. W. Korohoda, L. Rakoczy, and T. Walczak. On the control mechanism of protoplasmic streamings in the plasmodia of Myxomycetes. Acta Protozoologica, VII(29):363-373, 1970.

19. T. Ueda, K. Matsumoto, and Y. Kobatake. Spatial and temporal organization of intracellular adenine nucleotides and cyclic nucleotides in relation to rhythmic motility in physarum plasmodium. Experimental Cell Research, 162(2):486-494, February 1986.

20. A. C. H. Durham and E. B. Ridgway. Control of chemotaxis in physarum polycephalum. The Journal of Cell Biology, 69:218-223, 1976.

21. H. Miura and M. Yano. A model of organization of size invariant positional information in taxis of physarum plasmodium. Progress of Theoretical Physics, 100(2):235251, 1998.

22. Y. Miyake, S. Tabata, H. Murakami, M. Yano, and H. Shimizu. Environmentaldependent self-organization of positional information field in chemotaxis of physarum plasmodium. Journal of Theoretical Biology, 178:341-353, 1996.

23. T. Nakagaki, H. Yamada, and A. Toth. Intelligence: Maze-solving by an amoeboid organism. Nature, 407:470, 2000.

24. T. Nakagaki, R. Kobayashi, Y. Nishiura, and T. Ueda. Obtaining multiple separate food sources: behavioural intelligence in the physarum plasmodium. R. Soc. Proc.: Biol. Sci., 271(1554):2305-2310, 2004.

25. M. Aono. Personal communication, 2004.

26. S. Tsuda, M. Aono, and Y.-P. Gunji. Robust and emergent physarum logicalcomputing. BioSystems, 73:45-55, 2004.

27. K.-P. Zauner. From prescriptive programming of solid-state devices to orchestrated self-organisation of informed matter. In J.-P. Banâtre, J.-L. Giavitto, P. Fradet, and O. Michel, editors, Proceedings of UPP 2004, Unconventional Programming Paradigms, 15-17 September, Le Mont Saint-Michel, France, volume 3566 of LNCS, pages 47-55. Springer, 2005.

28. A. Takamtsu, T. Fujii, and I. Endo. Control of interaction strength in a network of the true slime mold by a microfabricated structure. BioSystems, 55:33-38, 2000.

29. S. Nomura. Symbolization of an object and its freedom in biological systems. PhD thesis, Kobe University, 2001.

30. T. Ueda, Y. Mori, T. Nakagaki, and Y. Kobatake. Action spectra for superoxide generation and UV and visible light photoavoidance in plasmodia of physarum polycephalum. Photochemistry and Photobiology, 48:705-709, 1988.

31. M. Conrad. Emergent computation through self-assembly. Nanobiology, 2:5-30, 1993.

32. Y.-P. Gunji, T. Takahashi, and M. Aono. Dynamical infomorphism: form of endoperspective. Chaos, Solitons and Fractals, 22:1077-1101, 2004. 